\title{
Prediction of BRCA1/2 mutation status in patients with ovarian cancer from a hospital-based cohort
}

Catharina E. Jacobi, $P h D^{1}$, Yvette van Ierland, $\mathrm{MSc}^{2}$, Christi J. van Asperen, $M D, P h D^{3}$, Eric Hallensleben, $M D, P h D^{6}$, Peter Devilee, $P h D^{2}$, Gert Jan Fleuren, $M D, P h D^{4}$, and Gemma G. Kenter, $M D, P h D^{5}$

\begin{abstract}
Purpose: To describe patient, tumor, and family histories of cancer in a hospital-based cohort of patients with ovarian cancer and to identify the predictive value of these characteristics for (non)carrying a BRCA1 or BRCA2 mutation. Methods: Women diagnosed with invasive ovarian cancer between 1999 and 2003 in the west region of The Netherlands and unselected for age at diagnosis or cancer family history were included. Information was gathered on patient and tumor characteristics; p53; HER-2/neu, and KI-67 protein-expression; BRCA1/2 mutations; and family histories of cancer. Prediction tests were constructed using multivariate analyses. Results: Our study included 85 women (mean age at diagnosis, 57.6 years; standard deviation, 11.0 years). Six of these women had been previously or concurrently diagnosed with another tumor. Of the ovarian cancers, $41(48.2 \%)$ were in an early stage (FIGO I or II). Five pathogenic mutations (6.1\%) and six unclassified variants (7.3\%) were identified in BRCA1/2; when the total sensitivity of the mutation scanning was taken into account, it was estimated to reflect seven pathogenic mutations (8.5\%) and eight unclassified variants (9.8\%). Sixty-nine women (81.2\%) had at least one relative with cancer. A personal history of breast cancer and a family history of breast, ovarian, or uterine/ endometrioid cancer were found to predict the presence of pathogenic mutations. Conclusion: As the combination of a personal history of breast cancer and a family history of breast, ovarian, or uterine/endometrioid cancer had good predictive value for the presence of a pathogenic BRCA1/2 mutation, the presented prediction test is a useful instrument to identify those women eligible for DNA testing. Genet Med 2007:9(3):173-179.
\end{abstract}

Key Words: prediction, histology, ovarian cancer, family history, BRCA1/2 mutation

\section{INTRODUCTION}

Ovarian cancer is the sixth most common cancer in women, accounting for 3 to $4 \%$ of all cancers in women. It represents approximately 25 to $30 \%$ of all cancers of the female genital organs $^{1}$ and is the most frequent cause of death from gynecological cancer. ${ }^{1}$ In the general population, the incidence of ovarian cancer is estimated at 1.5 per 1000 women per year, with an average age at diagnosis of 60 years. The total lifetime risk to develop ovarian cancer in the general population is 1 to $2 \%$, and the risk of dying of ovarian cancer is estimated at $0.7 \% .{ }^{1}$ Previous studies showed a crude overall 5-year survival rate of $39 \%$, with median survival of 32 months $^{2}$ and crude 5 -year survival rates of $82 \%, 57 \%, 28 \%$, and $10 \%$ for women with FIGO stage IC, II, III, and IV, respectively. ${ }^{3}$

\footnotetext{
Departments of ${ }^{1}$ Medical Decision Making, ${ }^{2}$ Human Genetics, ${ }^{3}$ Clinical Genetics, ${ }^{4}$ Pathology, and ${ }^{5}$ Gynecology, Leiden University Medical Center, Leiden; ${ }^{6}$ Gynecology, Groene Hart Ziekenhuis, Gouda, The Netherlands.

Dr. Catharina E. Jacobi, Department of Medical Decision Making (J10-S), Leiden University Medical Center, PO Box 9600, 2300 RC Leiden, The Netherlands. E-mail: C.E.Jacobi@ LUMC.NL and G.G.Kenter@LUMC.NL

Submitted for publication September 25, 2006.

Accepted for publication December 21, 2006.

The authors declare no conflict of interest.
}

DOI: 10.1097/GIM.0b013e318032e4ab
Genetic factors may increase the risk of ovarian cancer considerably. Mutations in the breast cancer susceptibility genes BRCA1 and BRCA2 are associated not only with high breast cancer risks, but also with increased risk of ovarian cancer. ${ }^{4-7}$ Estimates of the lifetime ovarian cancer risk in women vary from 22 to $51 \%$ for BRCA1 mutation carriers and from 4.1 to $18 \%$ for BRCA2 mutation carriers. ${ }^{6}$ Higher lifetime risks of developing ovarian cancer have been reported in multiple case families with breast and/or ovarian cancer and in Ashkenazi Jewish women who are mutation carriers. ${ }^{4,5,7}$ It has been shown that a pathogenic mutation in the BRCA1 or BRCA2 genes could be found in approximately $10 \%$ of all patients with ovarian cancer, ${ }^{8}$ which indicates that no BRCA1/2 mutations are identifiable in approximately $90 \%$ of patients with ovarian cancer.

As BRCA1 and BRCA2 mutations are associated not only with high ovarian cancer risks compared with the general population, but also with high risks of breast cancer, ${ }^{4-7}$ it is important to be able to select those patients who are likely to carry a BRCA1/2 mutation to provide DNA testing. Results of these tests are important not only for follow-up or surveillance strategies for the patient herself, but also for her relatives, both regarding breast cancer screening and possible preventive strategies on breasts or ovaries. In the present study, 85 patients with ovarian carcinoma unselected for age and family history 
of cancer were screened for the presence of BRCA1 and BRCA2 mutations. Our aim was to identify clinical or histopathological factors or family characteristics that can predict whether a patient with ovarian cancer is a carrier of a BRCA mutation.

\section{PATIENTS AND METHODS}

\section{Patients}

Patients with a primary diagnosis of epithelial ovarian cancer during the period of January 1999 to September 2003 were included in this study. Every woman with a primary diagnosis of ovarian cancer was asked to participate, but being diagnosed with a borderline tumor was considered an exclusion criterion. Eight different hospitals, all members of the Dutch Comprehensive Cancer Center West (IKW) and located in five cities in the west of The Netherlands (Leiden, Den Haag, Zoetermeer, Gouda, and Leidschendam) provided patients for this study. All patients gave written informed consent. This study was approved by the medical ethics committees of the participating hospitals. The group of participants was compared with the total population of the Dutch Comprehensive Cancer Center West (IKW) registered in the same period, and no differences in age, sex, and tumor characteristics were found. We may therefore conclude that our cohort of women is a representative sample of all women diagnosed with ovarian cancer in the same period.

\section{Patient and tumor characteristics}

As therapy, all patients underwent either a staging laparotomy according to the national guidelines or a debulking procedure, depending on the stage of disease. Surgical stage was classified according to FIGO criteria. ${ }^{9}$ The epithelial tumors were selected and classified following World Health Organization guidelines: $1=$ serous, $2=$ mucinous, $3=$ endometrioid, $4=$ clear cell, $5=$ undifferentiated, $6=$ extra-ovarian. Patients with unknown tumor type were excluded. After informed consent, a blood sample was taken for DNA analysis. Information concerning patient characteristics and family history of cancer was collected by questionnaires. Information was gathered on age at diagnosis, personal history of cancer, cancer among relatives in the first- (parents, siblings, and children) and seconddegree (grandparents, siblings of the parents, and grandchildren). If relatives had been diagnosed with cancer, their ages at diagnoses were also determined if possible. If no information was received on the presence of cancer in the family, the family history was classified as being absent. The family history of cancer was reported by the index patient only, without clinical confirmation. DNA and tumor blocks of the index patients were then analyzed.

\section{Mutation scanning of BRCA1 and BRCA2}

Genomic DNA was extracted from peripheral white blood cells using a salting-out procedure. ${ }^{10}$ The entire coding sequences of BRCA1 and BRCA2 were investigated by conformation-sensitive capillary electrophoresis (CSCE). ${ }^{11}$ Polymerase chain reaction (PCR) fragments were designed to amplify the coding exons plus $50 \mathrm{bp}$ of intronic sequence at either end of the exon. The maximal PCR fragment size was $400 \mathrm{bp}$, with multiple fragments covering the larger exons with a minimum of 100-bp overlap. One of the primers in each pair was labeled with FAM, HEX, or NED fluorescent dye (Applied Biosystems). The gene sequence of BRCA1 was divided into $31 \mathrm{PCR}$ fragments, and that of BRCA2 was divided into 48 fragments. PCR products were analyzed on an ABI3100 automated sequencer under specified running conditions. ${ }^{11}$ The trace files resulting from these runs were manually checked for the presence of aberrant peak patterns. In addition, all samples were screened specifically for the presence of the two Dutch founder gene rearrangements in BRCA1 by deletion junction PCR. ${ }^{12}$ When aberrant peak patterns were observed, the culprit gene fragment was re-amplified and sequenced.

As part of the treatment policy, patients with a highly affected family history were referred to the Department of Clinical Genetics. For those patients $(n=5)$, mutation testing of BRCA1 and BRCA2 was performed by the Laboratory of Clinical Gene Analysis using a combination of full-sequence analysis and denaturing gradient gel electrophoresis, together covering the entire coding regions of both genes, and multiple ligation-dependent probe amplification to detect large deletions/duplications in BRCA1. ${ }^{13}$

Sequence traces were analyzed using Mutation Surveyor 2.02 (Soft Genetics Inc.) and Chromas software (Technelysium). The mutations found were compared with the Breast Cancer Information Core (BIC) database and the Dutch mutation database of the Department of Clinical Genetics, Leiden University Medical Center.

The sensitivity of CSCE has been estimated to be approximately $80 \% .{ }^{11}$ PCR failure rates averaged $10 \%$; therefore, the overall sensitivity of the entire mutation analysis procedure is estimated at $72 \%$.

\section{Tumor tissue micro-array construction}

The patient's archival paraffin-embedded tumor blocks and corresponding hematoxylin and eosin (H\&E)-stained tissue sections were collected from the participating hospitals. If tissue sections were not available, fresh sections were cut from the donor blocks and stained with H\&E. The areas of highest tumor density were selected on the respective H\&E stained sections. Three tissue cores $(0.6 \mathrm{~mm}$ in diameter) were punched from the designated area using a biopsy needle and assembled into the TMA. ${ }^{14,15}$

\section{Immunohistochemistry}

Immunohistochemical staining of the tumor tissue was performed by using the streptavidin-biotin-peroxidase complex method (DAKO, Glostrup, Denmark) with the heat-induced antigen retrieval step. Control sections consisted of serial sections from the same blocks that were similarly processed during the experiment. Counterstaining was performed with hematoxylin. Immunoreactivity of p53, HER-2/neu, and KI-67 was quantitatively estimated for intensity $(1=$ weak, $2=$ nor- 
mal, $3=$ strong) and for percentage of positive tumor cells $(1=0-25 \%, 2=25-50 \%, 3=50-75 \%, 4=75-100 \%)$.

\section{Statistical analysis}

Univariate analyses were performed using Pearson's $\chi^{2}$ tests and Fisher's exact test for the categorical variables and using $t$ tests for the continuous variables.

To determine factors or characteristics that may predict the presence of a BRCA1/2 mutation, patients with pathogenic mutation (PM) in BRCA1 or BRCA2 were compared with those patients without such mutations (Comparison 1). As the study population was relatively small, no difference was made between carriers of a BRCA1 and carriers of a BRCA2 mutation. As mutations of the unclassified variance (UV) type may potentially be pathogenic, we re-performed the analyses comparing patients with a pathogenic mutation or an unclassified variant in BRCA1/2 with patients without such mutations (Comparison 2).

All characteristics that showed some association with mutation status in the univariate analyses $(P$ values $<0.100)$ were included into the multivariate stepwise logistic regression analyses. In this way, two multivariate analyses were performed.

Using the results of the multivariate regression analysis, we developed a prediction test for the above-mentioned comparisons. Sensitivity, specificity, and positive and negative predictive values of these tests were explored.

\section{RESULTS}

\section{Patients}

The study group consisted of 85 women diagnosed with ovarian cancer. The characteristics of the patients are presented in Table 1. On average, the patients were 57.6 years (SD $11.0 \mathrm{yr}$; range, $29-83 \mathrm{yr}$ ) at diagnosis. Of all the patients, 17 women $(20.0 \%)$ were younger than 50 years of age at diagnosis, and 8 of these were younger than 40 years of age at diagnosis ( $9.4 \%$ of total group). All women were Caucasian.

\section{Patient and tumor characteristics}

In six women, another tumor had been diagnosed previously or concurrently; three with breast cancer and three with uterine/endometrioid cancer. Regarding the FIGO classification of the tumors, $29(34.1 \%)$ were classified as stage I, 12 $(14.1 \%)$ as stage II, $38(44.7 \%)$ as stage III, and $6(7.1 \%)$ as stage IV.

Tumors of all types were found (Table 1), but most were of the serous type ( $n=37 ; 43.5 \%)$, followed by the endometrioid type $(n=22 ; 25.9 \%)$, then the mucinous type $(n=14 ; 16.5 \%)$.

\section{Family history}

In total, the 85 women reported 238 first- and second-degree family members with any type of cancer (mean, 2.80; range, $0-11)$. Forty-six patients $(54.1 \%)$ reported at least one first-degree relative with cancer; 23 women $(27.1 \%)$ had only second-degree relatives with cancer. No relatives with cancer were reported by only 16 women ( $18.8 \%)$. In total, 27 women $(31.8 \%)$ reported at least one first- or second-degree relative with breast or ovarian cancer: 17 women $(20.0 \%)$ had relatives with breast cancer, 4 women (4.7\%) had relatives with ovarian cancer, and 6 women $(7.1 \%)$ had relatives with breast cancer and relatives with ovarian cancer. Almost three quarters of the patients did not report relatives with breast or ovarian cancer $(n=58 ; 68.2 \%)$.

\section{Mutations in BRCA1 and BRCA2 genes}

In the study group, five PMs (three in BRCA1 and two in BRCA2) and six UVs (three in BRCA1 and three in BRCA2) were identified (Table 2). In 36 women, no variations in the BRCA1/2 genes were detected. In 35 patients, variations classified as polymorphisms were detected in the BRCA1/2 genes. In three patients, no results of the DNA tests could be reported, so these patients were excluded from these analyses. Taking the sensitivity of the mutation analysis (72\%) into account, one might expect that the true mutation rate that could be found in our study population should be seven PMs (8.5\%) and eight UVs (9.8\%); i.e., five PMs divided by $72 \%$ makes seven PMs, and six UVs divided by $72 \%$ makes eight UVs.

For analysis, the 5 patients with a PM were compared with 77 patients without such mutations. Additionally, the 11 patients with a PM or UV in BRCA1/2 were compared with the 71 patients without such mutations.

\section{Prediction of mutation status}

Comparison 1: pathogenic mutations versus nonpathogenic mutations

We were unable to detect statistically significant associations between the presence of a PM and age, FIGO staging, histological type, or any of the tumor markers (p53, HER-2/neu, or KI-67). Patients carrying a PM more often had a tumor of the serous type compared with the patients without PMs $(80.0 \%$, $n=4$ vs. $41.6 \%, n=32 ; P$ value 0.113 ), but this finding was not statistically significant. Moreover, these patients never had a tumor of the endometrioid histological type ( $0 \%$ vs. $27.3 \%$; $P$ value not significant). Patients with a PM more often had a personal history of breast cancer compared with patients without a PM ( $P$ value 0.009$)$. Regarding the family history of cancer, PM carriers more often had relatives with breast cancer (60.0\% vs. $22.1 \%$; $P$ value 0.091$)$ and relatives with ovarian cancer $(40.0 \%$ vs. $9.1 \%$; $P$ value 0.090$)$ compared with noncarriers, but this was not statistically significant. PM carriers more often had three or more relatives with breast or ovarian cancer $(40.0 \%$ vs. $3.9 \%, P$ value 0.028$)$.

We found that the combination of four variables [i.e., a personal history of breast cancer (yes $=2$, no $=0$ ), breast cancer in the family (yes $=1$, no $=0$ ), ovarian cancer in the family (yes $=1$, no $=0$ ), and uterine/endometrioid cancer among first-degree relatives (yes $=2$, no $=0$ )] could best predict the presence of a PM. Using a cutoff point of 2, this combination led to a sensitivity of $80 \%$ (four of five mutation carriers would 
Table 1

Characteristics of patients with ovarian cancer unselected for age or family history of cancer

\begin{tabular}{|c|c|c|c|c|c|c|c|}
\hline & $\mathrm{N}_{\max }^{\text {All }}=85$ & $\begin{array}{c}\mathrm{PM} \\
\mathrm{N}_{\max }=5\end{array}$ & $\begin{array}{c}\text { non-PM } \\
\mathrm{N}_{\max }=77\end{array}$ & $P$ value & $\begin{array}{l}\text { PM or UV } \\
\mathrm{N}_{\max }=11\end{array}$ & $\begin{array}{c}\text { non-PM/UV } \\
\mathrm{N}_{\max }=71\end{array}$ & $P$ value \\
\hline \multicolumn{8}{|c|}{$\begin{array}{l}\text { Patient characteristics } \\
\text { Age at diagnosis (yr) }\end{array}$} \\
\hline Mean (SD) & $57.6(11.0)$ & 61.9 (4.7) & $57.1(11.3)$ & NS & $51.8(13.1)$ & $58.2(10.6)$ & $<0.10$ \\
\hline$<50 \mathrm{yr}$ & $17(20.0)$ & $0(0.0)$ & $17(22.1)$ & NS & $4(36.4)$ & $13(18.3)$ & NS \\
\hline \multicolumn{8}{|c|}{ Tumor characteristics } \\
\hline \multicolumn{8}{|l|}{ FIGO stage } \\
\hline I & $29(34.1)$ & $1(20.0)$ & $27(35.1)$ & \multirow[t]{4}{*}{ NS } & $5(45.5)$ & $23(32.4)$ & \multirow[t]{4}{*}{ NS } \\
\hline II & $12(14.1)$ & $0(0.0)$ & $12(15.6)$ & & $0(0.0)$ & $12(16.9)$ & \\
\hline III & $38(44.7)$ & $3(60.0)$ & $33(42.9)$ & & $5(45.5)$ & $31(43.7)$ & \\
\hline IV & $6(7.1)$ & $1(20.0)$ & $5(6.5)$ & & $1(9.1)$ & $5(7.0)$ & \\
\hline \multicolumn{8}{|l|}{ Tumor type } \\
\hline Serous & $37(43.5)$ & $4(80.0)$ & $32(41.6)$ & \multirow[t]{6}{*}{ NS } & $6(54.5)$ & $30(42.3)$ & \multirow[t]{6}{*}{ NS } \\
\hline Mucinous & $14(16.5)$ & $0(0.0)$ & $13(16.9)$ & & $3(27.3)$ & $10(14.1)$ & \\
\hline Endometrioid & $22(25.9)$ & $0(0.0)$ & $21(27.3)$ & & $0(0.0)$ & $21(29.6)$ & \\
\hline Clear cell & $4(4.7)$ & $0(0.0)$ & $4(5.2)$ & & $1(9.1)$ & $3(4.2)$ & \\
\hline Undifferentiated & $3(3.5)$ & $0(0.0)$ & $3(3.9)$ & & $0(0.0)$ & $3(4.2)$ & \\
\hline Extraovarian & $5(5.9)$ & $1(20.0)$ & $4(5.2)$ & & $1(9.1)$ & $4(5.6)$ & \\
\hline \multicolumn{8}{|l|}{ Marker: p53 } \\
\hline Mean (SD) & $5.13(2.01)$ & $6.05(1.71)$ & $5.01(2.04)$ & NS & $5.19(2.08)$ & $5.06(2.04)$ & NS \\
\hline \multicolumn{8}{|l|}{ Her- $2 / \mathrm{Neu}$} \\
\hline Mean (SD) & $5.55(0.90)$ & $5.83(0.24)$ & $5.54(0.94)$ & NS & $5.81(0.56)$ & $5.52(0.96)$ & NS \\
\hline \multicolumn{8}{|l|}{ KI-67 } \\
\hline Mean (SD) & $4.74(1.17)$ & $5.59(1.44)$ & $4.69(1.16)$ & NS & $4.89(1.32)$ & $4.72(1.17)$ & NS \\
\hline \multicolumn{8}{|l|}{ KI-67-cells } \\
\hline Mean (SD) & $1.86(0.99)$ & $2.59(1.44)$ & $1.82(0.97)$ & NS & $2.00(1.18)$ & $1.85(0.98)$ & NS \\
\hline \multicolumn{8}{|l|}{ Personal history } \\
\hline \multicolumn{8}{|l|}{ Cancer } \\
\hline Yes & $6(7.1)$ & $2(40.0)$ & $4(5.2)$ & $<0.05$ & $2(18.2)$ & $4(5.6)$ & NS \\
\hline \multicolumn{8}{|l|}{ Breast cancer } \\
\hline Yes & $3(3.5)$ & $2(40.0)$ & $1(1.3)$ & $<0.01$ & $2(18.2)$ & $1(1.4)$ & $<0.05$ \\
\hline \multicolumn{8}{|l|}{ Family history } \\
\hline \multicolumn{8}{|l|}{ Cancer } \\
\hline Mean (SD) & $2.80(2.51)$ & $4.80(3.49)$ & $2.53(2.33)$ & $<0.05$ & $3.82(2.89)$ & $2.49(2.35)$ & $<0.10$ \\
\hline \multicolumn{8}{|l|}{ Cancer } \\
\hline Yes & 69 (81.2) & $4(80.0)$ & $62(80.5)$ & NS & $9(81.8)$ & $57(80.3)$ & NS \\
\hline \multicolumn{8}{|l|}{ Breast cancer } \\
\hline Mean (SD) & $0.41(0.78)$ & $1.40(1.52)$ & $0.30(0.63)$ & $<0.001$ & $1.18(1.33)$ & $0.24(0.52)$ & $<0.05$ \\
\hline \multicolumn{8}{|l|}{ Breast cancer } \\
\hline Yes & $23(27.1)$ & $3(60.0)$ & $17(22.1)$ & $<0.10$ & $6(54.5)$ & $14(19.7)$ & $<0.05$ \\
\hline \multicolumn{8}{|l|}{ Ovarian cancer } \\
\hline Mean (SD) & $0.14(0.41)$ & $0.40(0.55)$ & $0.10(0.35)$ & NS & $0.18(0.41)$ & $0.11(0.36)$ & NS \\
\hline Ovarian cancer & & & & & & & \\
\hline Yes & $10(11.8)$ & $2(40.0)$ & $7(9.1)$ & $<0.10$ & $2(18.2)$ & $7(9.9)$ & NS \\
\hline Breast or ovarian ca & & & & & & & \\
\hline Yes & $27(31.8)$ & $3(60.0)$ & $21(27.3)$ & NS & $6(54.5)$ & $18(25.4)$ & $<0.10$ \\
\hline $\begin{array}{l}\text { Breast or ovarian or } \\
\text { uterine/endom } \\
\text { cancer }\end{array}$ & & & & & & & \\
\hline Yes & $36(42.4)$ & $4(80.0)$ & $29(37.7)$ & $<0.10$ & $7(63.6)$ & $26(36.6)$ & $<0.10$ \\
\hline Breast and prostate & & & & & & & \\
\hline Yes & $8(9.4)$ & $1(20.0)$ & $6(7.8)$ & NS & $2(18.2)$ & $5(7.0)$ & NS \\
\hline
\end{tabular}

$N=85$.

Values are $n(\%)$ unless stated otherwise.

$\mathrm{N}_{\max }$, maximum number of patients; PM, pathogenic mutation BRCA1/2; UV, unclassified variant mutation BRCA1/2; NS, not statistically significant $(P>0.100)$. 
Table 2

Mutations found in the BRCA1 and BRCA2 genes

\begin{tabular}{|c|c|c|c|}
\hline Exon/intron & NT change $^{a}$ & $\begin{array}{l}\text { Amino acid } \\
\text { change }\end{array}$ & Variation type \\
\hline \multicolumn{4}{|l|}{ BRCA1 } \\
\hline \multicolumn{4}{|c|}{ Pathogenic mutation } \\
\hline $11^{b}$ & 2138delA & & Frameshift \\
\hline 11 & 2804delAA & & Frameshift \\
\hline \multicolumn{4}{|c|}{ Unclassified variant } \\
\hline 8 & $655 \mathrm{~A}>\mathrm{G}$ & Y179C & Missense \\
\hline 11 & $3238 \mathrm{G}>\mathrm{A}$ & S1040N & Missense \\
\hline 14 & IVS13-10C $>\mathrm{T}$ & & Intronic variation \\
\hline \multicolumn{4}{|l|}{ BRCA2 } \\
\hline \multicolumn{4}{|c|}{ Pathogenic mutation } \\
\hline 11 & 6648insA & & Frameshift \\
\hline 11 & $6785 \mathrm{C}>\mathrm{A}$ & S2186X & Nonsense \\
\hline \multicolumn{4}{|c|}{ Unclassified variant } \\
\hline 18 & $8369 A>G$ & Q2714R & Missense \\
\hline 18 & $8415 \mathrm{G}>\mathrm{T}$ & K2729N & Missense \\
\hline 22 & $9079 \mathrm{G}>\mathrm{A}$ & A2951T & Missense \\
\hline
\end{tabular}

NT, nucleotide change.

${ }^{a}$ According to the BIC nomenclature.

${ }^{b}$ This mutation was identified twice in this study.

be found) and a specificity of $91 \%$ (70 of the 77 non-carriers would not be selected for genetic testing) (Table 3 ). These data show that a patient with a positive test outcome is 25 times more likely to be carrying a PM compared with patients with a negative test outcome ( 4 of 11 vs. 1 of 70). These data show that, given a negative test outcome, the patient is unlikely to be a mutation carrier (i.e., 1 of 70, thus $1.4 \%$ ).

\section{Comparison 2: pathogenic mutations and unclassified} variants versus non-carriers

Associations between the presence of a PM or UV and all characteristics are shown in Table 1.
The combination of three variables [i.e., a personal history of breast cancer (yes $=1 ;$ no $=0$ ), the presence of breast cancer in the family (yes $=1 ;$ no $=0$ ), and a diagnosis age of 40 years or younger (yes $=1 ;$ no $=0)$ ] could best predict the presence of a PM or UV. This combination led to a sensitivity of $82 \%$ (9 of $11 \mathrm{PM} / \mathrm{UV}$ carriers would be found) and a specificity of $80 \%$ (57 of the 71 non-carriers would not be selected for genetic testing) (Table 3). These data show that a patient with a positive outcome on this second test is almost 12 times more likely to be carrying a PM or UV compared with patients with a negative test outcome ( 9 of 23 vs. 2 of 59). These data also show that, given a negative test outcome, a patient is unlikely to be a carrier of a PM or UV (i.e., 2 of 59, thus 3.4\%).

\section{DISCUSSION}

In this article, we describe a hospital-based cohort of patients with ovarian cancer unselected for age or family history. We present characteristics of these patients and of their families that are associated with the presence of mutations in the BRCA1/2 genes. We found that a personal history of breast cancer and the presence of breast and ovarian cancer or uterine/endometrioid cancer in the family are highly predictive of the presence of a PM, whereas a personal history of family history of breast cancer or young age at diagnosis was highly predictive for the presence of a PM or an UV in the BRCA1/2 genes. Although these characteristics are predictive for the presence of BRCA1/2 mutations, it always remains a possibility that a patient can have no personal or familial characteristics associated with BRCA1/2 mutations and still carry such a PM or UV. However, when the outcomes of the presented prediction tests are negative, it is unlikely that the patient is a carrier of a pathogenic mutation or UV in BRCA1/2 (varying from 1.4 to $3.4 \%$ ).

We found that $6.1 \%(n=5)$ of our patients were carriers of a PM in the BRCA1/2 genes. The frequency of PMs in BRCA1 $(n=3 ; 3.7 \%)$ was higher than in BRCA2 $(n=2 ; 2.4 \%)$. A difference in mutation prevalence is to be expected when looking at the penetrance of ovarian cancer of pathogenic mutations in BRCA1 versus BRCA2. ${ }^{6}$ In addition, we identified six

Table 3

Sensitivity, specificity, and positive and negative predictive values of combinations of variables to predict the presence or absence of a mutation

\begin{tabular}{|c|c|c|c|c|c|c|}
\hline & Prediction variables & Cutoff value & $\mathrm{Se}$ & Sp & PPV & NPV \\
\hline & Presence of $\mathrm{BC}$ in family $($ yes $=1 ;$ no $=0$ ) & & & & & \\
\hline & Presence of OV in family (yes $=1 ;$ no $=0$ ) & & & & & \\
\hline & Presence of UT in first-degree relatives (yes $=2 ;$ no $=0$ ) & & & & & \\
\hline \multirow{2}{*}{$\begin{array}{l}\text { Comparison 2: PM or UV vs. } \\
\text { non-PM/UV }\end{array}$} & Personal history of $\mathrm{BC}($ yes $=1 ;$ no $=0)$ & 1 or more & $82 \%$ & $80 \%$ & $39 \%$ & $97 \%$ \\
\hline & Diagnosis age $<40$ yrs $($ yes $=1$; no $=0)$ & & & & & \\
\hline
\end{tabular}

$N=85$.

PM, pathogenic mutation in BRCA1/2; UV, unclassified variant mutation in BRCA1/2; Se, sensitivity; Sp, specificity; PPV, positive predictive value; NPV, negative predictive value; $\mathrm{BC}$, breast cancer; OV, ovarian cancer; UT, uterine/endometrioid cancer. 
UVs (three in BRCA1 and three in BRCA2), of which the relation with disease risk is unclear.

The mutation frequency of $6.1 \%$ for BRCA1/2 as identified in our study seems to be an underestimation. This low estimate is probably the result of the failure of the mutation screening of some gene fragments and the sensitivity of the techniques. As the sensitivity of CSCE has been estimated to be approximately $80 \%{ }^{11}$ and the PCR failure rates were on average $10 \%$, this frequency should be approximately $8.5 \%$. Furthermore, it might be that some of the detected mutations, which were classified as UV or polymorphisms, are of clinical relevance as well. A meta-analysis of six studies ${ }^{16-21}$ of an unselected series of ovarian cancers indicated that $5.7 \%$ (70 of 1236) of all ovarian cancers are associated with germline mutations of BRCA1, whereas four such studies ${ }^{20-23}$ indicated that $3.8 \%$ (28 of 738 ) of all ovarian cancers are associated with germline mutations in BRCA2. ${ }^{8}$ Our finding of an estimated mutation frequency of $8.5 \%$ is in the range of this meta-analysis. However, the combined figure of $9.5 \%$ of the meta-analysis is likely to be an underestimate, as all of the included studies utilized indirect methods for mutation screening. ${ }^{8}$ This may be a problem in the current study as well. In more recent studies, higher mutation frequencies were found. ${ }^{6,24,25}$ Moreover, in our study, under-reporting of mutation frequency may have taken place. For instance, no DNA test results were available for three patients. These three patients all reported a family history of breast cancer; one also a family history of ovarian cancer but no personal history of cancer or family histories of uterine/endometrioid cancer. Based on our own prediction model, one of these three patients should receive BRCA mutation scanning, leading to a $36 \%$ chance of finding a PM.

The mean age at diagnosis of our patients ranged from 29 to 83 years, with a mean of 57.6 years. This is comparable to other studies. ${ }^{26-28}$ The carriers of a PM had a slightly higher age at diagnosis compared with the non-carriers (61.9 vs. $57.1 \mathrm{yr}$ ), but this difference was not statistically significant. Similar results have been reported in previous studies, showing that the mean age of onset for ovarian cancer was comparable in mutation-positive and -negative families..$^{21,29,30}$ Others have reported a younger age at diagnosis for BRCA1 mutation families, compared with families without a mutation, whereas no difference was found for BRCA2 mutation carriers. ${ }^{31}$ In our cohort, patients carrying an UV were significantly younger at diagnosis compared with all other patients (43.4 vs. $58.5 \mathrm{yr}$, $P=0.001)$.

We were unable to find any association between mutation status and the expression of tumor markers such as p53, Her2neu, and K-67. This result is comparable to those of previous studies. ${ }^{8,32}$ we also found no associations between FIGO stage or histological type and mutation status, although most PM carriers had a tumor of the serous type (80\%). Previous studies have reported that no mucinous tumors were found in BRCA mutation-positive families..$^{21,33}$ Others, however, found some carriers of BRCA1/2 mutations to be diagnosed with mucinous tumors. ${ }^{34}$ We found that 14 patients had a mucinous tumor; 3 of these patients were carriers of UVs in BRCA1 or
BRCA2, but no patients with a mucinous tumor type were found to be PM carriers.

We found that the presence of breast and/or ovarian cancer clearly differed between the families of carrier and non-carriers, especially for breast cancer. The average number of firstand second-degree relatives with ovarian cancer was 0.40 for carriers of a PM and 0.10 for the patients without such mutations. The average number of relatives with breast cancer was 1.40 for PM carriers, 1.18 for PM/UV carriers, and 0.24 for the remaining patients. From the literature, it is known that the extent of family history of ovarian and breast cancer is strongly predictive of a BRCA mutation, ${ }^{26,35}$ which is in line with our PM prediction test. Studies have reported that no PMs were detected among patients who reported only one first- or second-degree relative with ovarian cancer. ${ }^{36} \mathrm{~A}$ large English follow-up study reported the ovarian cancer relative risk for families with more than two cases of ovarian cancer in the first degree to be 17.60 compared with the two-case only families. ${ }^{37}$ Furthermore, it has been proposed that testing for predisposing mutations is likely to be justified for any family in which two or more close relatives have epithelial ovarian cancer..$^{38}$ In our population, however, ovarian cancer was not always present in the family of PM carriers. Our results show that a positive family history of breast cancer, ovarian cancer, or uterine/endometrioid cancer or a personal history of breast cancer is a good predictor for the presence of a BRCA pathogenic mutation. It must be noted, however, that the reported diagnosis of uterine/endometrioid cancer in relatives of the index patients may be cases of misclassified ovarian cancer, as the family history was only reported by the index patient without clinical confirmation.

Mutations in the BRCA1/2 genes predispose women to hereditary breast and ovarian cancer syndrome and increase the lifetime risk of developing breast and/or ovarian cancer. ${ }^{4-7}$ It is estimated that at least $10 \%$ of all epithelial ovarian cancers are hereditary, with the BRCA genes contributing to at least 90\% of these cases and small percentages attributable to other genes, like HNPCC ${ }^{39}$ and perhaps a yet to be discovered susceptibility gene. ${ }^{8}$ As germline BRCA1/2 mutations are relatively uncommon among patients with ovarian cancer in the general population, it is very important to identify risk factors that can predict the likelihood of finding these mutations to identify mutation carriers. These actions should be undertaken to counsel mutation carriers and their high-risk family members adequately and to offer DNA testing and surveillance to family members. ${ }^{26,40}$ In this study, we propose a mutation prediction test to identify those patients with a high probability of carrying a pathogenic mutation or unclassified variant in the BRCA1/2 genes (Table 3 ): a combined family history of breast and ovarian cancer, or uterine/endometrioid cancer in firstdegree relatives, or a personal history of breast cancer provides an $80 \%$ sensitivity of the presence of a pathogenic BRCA1/2 mutation. To be able to use the presented tests, it is necessary to ask each patient for a complete cancer family history.

In summary, BRCA1 and BRCA2 germline mutations were detected in $6.1 \%$ of the patients with ovarian cancer in this 
study population, which is estimated to reflect a frequency of $8.5 \%$. Because ovarian cancer is a serious disease and DNA testing of all ovarian cancer patients is not cost-effective, it is important to identify those women who have a high probability of carrying a pathogenic BRCA1 or BRCA 2 mutation. To identify these patients, it is necessary to ask for a complete cancer family history from every ovarian cancer patient. If the patient has a personal history of breast cancer, or if she has relatives with both breast and with ovarian cancer, or if she has first-degree relatives with uterine/endometrioid cancer, she should be offered DNA testing of BRCA1/2 mutations. When studying a patient's family history, it should be remembered that ovarian cancer in combination with other tumors, such as colon cancer, may suggest the presence of other types of mutations, such as HNPCC.

\section{ACKNOWLEDGMENTS}

We thank all the gynecologists who participated by including their patients in this study. Moreover, we thank G.M.C. Ranke for data management.

\section{References}

1. Visser O, Siesling S, van Dijck JAAM (Eds.). Incidence of cancer in the Netherlands 1999/2000. Utrecht (NL): Association of Comprehensive Cancer Centres. The Netherlands, 2003.

2. Tingulstad S, Skjeldestad FE, Halvorsen TB, Hagen B. Survival and prognostic factors in patients with ovarian cancer. Obstet Gynecol 2003;101(5 Pt 1):885-891.

3. O’Malley CD, Cress RD, Campleman SL, Leiserowitz GS. Survival of Californian women with epithelial ovarian cancer, 1994-1996: a population-based study. Gynecol Oncol 2003;91:608-615.

4. Easton DF, Ford D, Bishop DT. Breast and ovarian cancer incidence in BRCA1mutation carriers: breast cancer linkage consortium. Am J Hum Genet 1995;56:265271.

5. Ford D, Easton DF, Stratton M, Narod S, et al. Genetic heterogeneity and penetrance analysis of the BRCA1 and BRCA2 genes in breast cancer families. The Breast Cancer Linkage Consortium. Am J Hum Genet 1998;62:676-689.

6. Antoniou A, Pharoah PD, Narod S, Risch HA, et al. Average risks of breast and ovarian cancer associated with BRCA1 or BRCA2 mutations detected in case series unselected for family history: a combined analysis of 22 studies. Am J Hum Genet 2003;72:1117-1130.

7. King MC, Marks JH, Mandell JB. Breast and ovarian cancer risks due to inherited mutations in BRCA1 and BRCA2. Science 2003;302:643-646.

8. Boyd J. Hereditary ovarian cancer: what we know. Gynecol Oncol 2003;88(1 Pt 2): S8-S10.

9. BC Cancer Agency. FIGO classification criteria of the ovaries. http://www. bccancer.bc.ca/HPI/CancerManagementGuidelines/Gynecology/OvaryEpithelial/ Staging.htm Accessed July 31, 2006.

10. Miller SA, Dykes DD, Polesky, HF. A simple salting out procedure for extracting DNA from human nucleated cells. Nucleic Acids Res 1988;16:1215.

11. Davies H, Dicks E, Stephens P, Cox C, et al. High throughput DNA sequence variant detection by conformation sensitive capillary electrophoresis and automated peak comparison. Genomics 2006;87:427-432.

12. Petrij-Bosch A, Peelen, T, van Vliet M, van Eijk R, et al. BRCAl genomic deletions are major founder mutations in Dutch breast cancer patients. Nat Genet 1997;17: 341-345.

13. Hogervorst FB, Nederlof PM, Gille JJ, McElgunn CJ, et al. Large genomic deletions and duplications in the BRCA1 gene identified by a novel quantitative method. Cancer Res 2003;63:1449-1453.
14. Kononen J, Bubendorf L, Kallioniemi A, Barlund M, et al. Tissue microarrays for high-throughput molecular profiling of tumor specimens. Nat Med 1998;4:844847.

15. Oldenburg RA, Kroeze-Jansema K, Meijers-Heijboer H, van Asperen CJ, et al. Characterization of familial non-BRCA1/2 breast tumors by loss of heterozygosity and immunophenotyping. Clin Cancer Res 2006;12:1693-1700.

16. Takahashi H, Behbakht K, McGovern PE, Chiu HC, et al. Mutation analysis of the BRCA1 gene in ovarian cancers. Cancer Res 1995;55:2998-3002.

17. Matsushima M, Kobayashi K, Emi M, Saito H, et al. Mutation analysis of the BRCA gene in 76 Japanese ovarian cancer patients: four germline mutations, but no evidence of somatic mutation. Hum Mol Genet 1995;4:1953-1956.

18. Stratton JF, Gayther SA, Russell P, Dearden J, et al. Contribution of BRCA1 mutations to ovarian cancer. N Engl J Med 1997;336:1125-1130.

19. Berchuck A, Heron KA, Carney ME, Lancaster JM, et al. Frequency of germline and somatic BRCAl mutations in ovarian cancer. Clin Cancer Res 1998;4:2433-2437.

20. Khoo US, Ngan HY, Cheung AN, Chan KY, et al. Mutational Analysis of BRCA1 and BRCA2 genes in Chinese ovarian cancer identifies 6 novel germline mutations. Hum Mutat 2000;16:88-89.

21. Risch HA, McLaughlin JR, Cole DE, Rosen B, et al. Prevalence and penetrance of germline BRCA1 and BRCA2 mutations in a population series of 649 women with ovarian cancer. Am J Hum Genet 2001;68:700-710.

22. Takahashi H, Chiu HC, Bandera CA, Behbakht K, et al. Mutations of the BRCA2 gene in ovarian carcinomas. Cancer Res 1996;56:2738-2741.

23. Foster KA, Harrington P, Kerr J, Russell P, et al. Somatic and germline mutations of the BRCA2 gene in sporadic ovarian cancer. Cancer Res 1996;56:3622-3625.

24. Pal T, Permuth-Wey J, Betts JA, Krischer JP, et al. BRCA1 and BRCA2 mutations account for a large proportion of ovarian carcinoma cases. Cancer 2005; 104:28072816.

25. Chen S, Iversen ES, Friebel T, Finkelstein D, et al. Characterization of BRCAl and BRCA2 mutations in a large United States sample. J Clin Oncol 2006;24:863-871.

26. Sarantaus L, Vahteristo P, Bloom E, Tamminen A, et al. BRCA1 and BRCA2 mutations among 233 unselected Finnish ovarian carcinoma patients. Eur J Hum Genet 2001;9:424-430.

27. Moslehi R, Chu W, Karlan B, Fishman D, et al. BRCA1 and BRCA2 mutation analysis of 208 Ashkenazi Jewish women with ovarian cancer. Am J Hum Genet 2000;66: 1259-1272.

28. Anton-Culver H, Cohen PF, Gildea ME, Ziogas A. Characteristics of BRCA1 mutations in a population-based case series of breast and ovarian cancer. Eur J Cancer 2000;36:1200-1208.

29. Claes K, Poppe B, Coene I, Paepe AD, et al. BRCA1 and BRCA2 germline mutation spectrum and frequencies in Belgian breast/ovarian cancer families. $\mathrm{Br} \mathrm{J}$ Cancer 2004;90:1244-1251.

30. Frank TS, Deffenbaugh AM, Reid JE, Hulick M, et al. Clinical characteristics of individuals with germline mutations in BRCA1 and BRCA2: analysis of 10,000 individuals. J Clin Oncol 2002;20:1480-1490.

31. Gayther SA, Russell P, Harrington P, Antoniou AC, et al. The contribution of germline BRCA1 and BRCA2 mutations to familial ovarian cancer: no evidence for other ovarian cancer-susceptibility genes. Am J Hum Genet 1999;65:1021-1029.

32. Zweemer RP, Shaw PA, Verheijen RM, Ryan A, et al. Accumulation of P53 protein is frequent in ovarian cancers associated with BRCA1 and BRCA2 germline mutations. J Clin Pathol 1999;52:372-375.

33. Werness BA, Ramus SJ, DiCioccio RA, Whittemore AS, et al. Histopathology, FIGO stage, and BRCA mutation status of ovarian cancers from the Gilda Radner Familial Ovarian Cancer Registry. Int J Gynecol Pathol 2004;23:29-34.

34. Lakhani SR, Manek S, Penault-Llorca F, Flanagan A, et al. Pathology of ovarian cancers in BRCA1 and BRCA2 carriers. Clin Cancer Res 2004;10:2473-2481.

35. Claus EB, Schildkraut JM, Thompson WD, Risch NJ. The genetic attributable risk of breast and ovarian cancer. Cancer 1996;77:2318-2324.

36. Russell PA, Pharoah PD, De Foy K, Ramus SJ, et al. Frequent loss of BRCA1 MRNA and protein expression in sporadic ovarian cancers. Int J Cancer 2000;87:317-321.

37. Rubin SC. BRCA-related ovarian carcinoma. Cancer 2003;97:2127-2129.

38. Puget N, Torchard D, Serova-Sinilnikova OM, Lynch HT, et al. A 1-Kb Alu-mediated germ-line deletion removing BRCA1 exon 17. Cancer Res 1997;57:828-831.

39. Malander S, Rambech E, Kristoffersson U, Halvarsson B, et al. The contribution of the hereditary nonpolyposis colorectal cancer syndrome to the development of ovarian cancer. Gynecol Oncol 2006;101:238-243.

40. Cannistra SA. Cancer of the ovary. N Engl J Med 2004;351:2519-2529. 